PROCEEDINGS OF THE

AMERICAN MATHEMATICAL SOCIETY

Volume 140, Number 7, July 2012, Pages 2319-2332

S 0002-9939(2011)11055-4

Article electronically published on October 3, 2011

\title{
ENTIRE FUNCTIONS OF FINITE ORDER AS SOLUTIONS TO CERTAIN COMPLEX LINEAR DIFFERENTIAL EQUATIONS
}

\author{
N. ANGHEL \\ (Communicated by Walter Van Assche)
}

\begin{abstract}
When is an entire function of finite order a solution to a complex 2nd order homogeneous linear differential equation with polynomial coefficients? In this paper we will give two (equivalent) answers to this question. The starting point of both answers is the Hadamard product representation of a given entire function of finite order. While the first answer involves certain Stieltjes-like relations associated to the function, the second one requires the vanishing of all but finitely many suitable expressions constructed via the Gil' sums of the zeros of the function. Applications of these results will also be given, most notably to the spectral theory of one-dimensional Schrödinger operators with polynomial potentials.
\end{abstract}

\section{INTRODUCTION}

Basic results such as the existence and uniqueness of solutions for initial value problems in differential equations and analytic continuation in complex analysis team up to show that all the solutions of a general complex 2nd order homogeneous linear differential equation

$$
y^{\prime \prime}(z)=u(z) y^{\prime}(z)+v(z) y(z), \quad z \in \mathbf{C}, u(z), v(z), \text { entire functions, }
$$

are entire functions. It is known, due to Nikolaus $\mathbb{N}, \mathrm{L}$, that a given entire function $f(z)$ is a solution to an equation of type (1) if and only if all the zeros of $f$, if any, are simple. Moreover, a classical result due to Frei and Wittich [F, W, L, states that all the solutions of (11) are entire functions of finite order if and only if $u(z)$ and $v(z)$ are polynomials. It is then natural to ask under what circumstances a given entire function of finite order is a solution to equation (11) with polynomial coefficients. Answers to this question, which naturally showcase the interplay between complex analysis and differential equations, are expected to shed new light on some important related problems such as the spectral theory of the one-dimensional Schrödinger operators with polynomial potentials.

For the convenience of the reader and in order to establish necessary notation, we gather here the needed facts regarding the entire functions of finite order. A very readable account of these matters can be found in $\mathrm{As}$.

Received by the editors September 29, 2010 and, in revised form, February 4, 2011.

2010 Mathematics Subject Classification. Primary 30D15, 34M05; Secondary 33C10, 34L40.

Key words and phrases. Complex differential equations, polynomial coefficients, entire functions, finite order, zeros, Stieltjes relations, Gil' sums, Schrödinger operators.

(C)2011 American Mathematical Society Reverts to public domain 28 years from publication 
Recall that a function $f(z)$ represented as a power series about $z=0$ by $\sum_{i=0}^{\infty} c_{i} z^{i}$, $c_{i} \in \mathbf{C}$, is entire if and only if $\lim _{i \rightarrow \infty} \sqrt[i]{\left|c_{i}\right|}=0 . f(z)$ is said to have finite order (of growth at infinity) if there is $\alpha>0$ such that

$$
|f(z)| \leq e^{|z|^{\alpha}}, \quad|z| \text { large enough, }
$$

and then the order of $f, \rho(f)$, is the infimum of all $\alpha$ 's satisfying equation (2). One has $\rho(f)=\limsup _{i \rightarrow \infty} \frac{i \ln i}{\ln \left|\frac{1}{c_{i}}\right|}$ As. The non-zero zeros, if any, of an entire function of finite order, $a_{1}, a_{2}, \ldots$, repeated according to multiplicity and labeled such that $0<\left|a_{1}\right| \leq\left|a_{2}\right| \leq \ldots$, have the property $[\mathrm{As}$ that

$$
\sum_{i} \frac{1}{a_{i}^{\alpha}}<\infty, \quad \alpha>\rho(f)
$$

Consequently, if $\mu \geq 0$ is the exponent of convergence $\lambda\left(\frac{1}{f}\right)$ for the zeros of $f$, namely the least non-negative real number such that for $\alpha>\mu, \sum_{i} \frac{1}{\left|a_{i}\right|^{\alpha}}<\infty$, then $\mu \leq \rho(f)$. Let $m$ be the non-negative integer associated to $\mu=\lambda\left(\frac{1}{f}\right)$, defined by

$$
m= \begin{cases}{[\mu],} & \text { if } \mu \text { is not an integer, } \\ \mu, & \text { if } \mu \text { is an integer and } \sum_{i} \frac{1}{\left|a_{i}\right|^{\mu}}=\infty, \\ \mu-1, & \text { if } \mu \text { is a positive integer and } \sum_{i} \frac{1}{\left|a_{i}\right|^{\mu}}<\infty, \\ 0, & \text { if }\left(a_{i}\right)_{i} \text { is a finite set. }\end{cases}
$$

The Hadamard product representation for an entire function of finite order $\mathrm{As}$. then asserts that there is a polynomial $q(z), \operatorname{deg}(q) \leq \rho(f)$, such that for every $z \in \mathbf{C}$,

$$
f(z)=e^{q(z)} z^{\epsilon} \prod_{i} E_{m}\left(\frac{z}{a_{i}}\right),
$$

where $\epsilon$ is the multiplicity of 0 as a zero of $f$, a non-negative integer, and $E_{n}(z)$ is the $n$th Weierstrass elementary factor defined by

$$
E_{n}(z)= \begin{cases}1-z, & \text { if } n=0, \\ (1-z) e^{\left(z+\frac{z^{2}}{2}+\cdots+\frac{z^{n}}{n}\right)}, & \text { if } n \geq 1 .\end{cases}
$$

The convergence of the product in equation (4) is guaranteed, in the usual way, by the absolute and uniform convergence on compact subsets of $\mathbf{C}$ of the series

$$
\sum_{i}\left(E_{m}\left(\frac{z}{a_{i}}\right)-1\right)
$$

the latter convergence being ensured, following classical work of Weierstrass, by the very definition of the exponent of convergence $\mu=\lambda\left(\frac{1}{f}\right)$ of the zeros of $f$ and 
its associated non-negative integer $m$. In fact, the convergence of many (infinite) products and series below will be a result of similar arguments, and as such will be implemented without further elaboration.

\section{Our Results}

We are now ready to state and prove our main results.

Theorem A. Assume that $f(z) \not \equiv 0$ is an entire function of finite order with nonzero zeros $a_{1}, a_{2}, \ldots$, if any, listed according to multiplicity and in non-decreasing order of their absolute values. Denote by $\mu$ the exponent of convergence of $\left(a_{i}\right)_{i}$ and by $m$ its associated non-negative integer ( $c f$. equation (3)). Then $f(z)$ is a solution to a differential equation $y^{\prime \prime}(z)=u(z) y^{\prime}(z)+v(z) y(z)$ with polynomial coefficients $u(z)$ and $v(z)$ if and only if all the zeros of $f(z)$ are simple (in particular, $\epsilon=0$ or 1) and there is some polynomial $p(z)$ such that the following Stieltjes-type relations hold true:

$$
p^{\prime}\left(a_{k}\right)=\sum_{i, i \neq k}\left(\frac{a_{k}}{a_{i}}\right)^{m} \frac{1}{a_{i}-a_{k}}-\frac{m+\epsilon}{a_{k}}, \quad k=1,2, \ldots
$$

and

$$
\epsilon p^{\prime}(0)= \begin{cases}\epsilon \sum_{i} \frac{1}{a_{i}}, & \text { if } m=0 \text { and }\left(a_{i}\right)_{i} \text { is non-empty } \\ 0, & \text { otherwise. }\end{cases}
$$

Moreover, $p(z)=q(z)-\frac{1}{2} \int u(z)$, where $q(z)$ is the polynomial appearing in the Hadamard product representation (4) of $f(z)$, and

$$
\rho\left(e^{-1 / 2 \int u} f\right)=\frac{\operatorname{deg}\left(\frac{1}{4} u^{2}+v\right)+2}{2} .
$$

In the particular case $u(z) \equiv 0, p(z)=q(z)$ and $\rho(f)=\frac{\operatorname{deg}(v)+2}{2}$.

Proof. By the uniqueness property of solutions of initial value differential equations it is clear that all the zeros of a non-trivial solution of an equation

$$
y^{\prime \prime}(z)=u(z) y^{\prime}(z)+v(z) y(z), \quad u(z), v(z) \text { polynomial functions, }
$$

are simple. A simple calculation now shows that $f(z)$ is a solution to the differential equation (8) if and only if $e^{-1 / 2 \int u(z)} f(z)$ is a solution to the differential equation

$$
y^{\prime \prime}(z)=\left(\frac{1}{4} u^{2}(z)-\frac{1}{2} u^{\prime}(z)+v(z)\right) y(z) .
$$

Since the transformed solution and differential equation preserve the polynomial aspect of our problem and of the Hadamard product representation, there is no loss of generality in proving the theorem in the particular case $u(z) \equiv 0$, when the polynomial $p(z)$ becomes $q(z)$.

Assume now, abstractly, that $g(z)$ is a non-trivial entire function with only simple zeros and that $g(z)=\prod_{j} g_{j}(z)$, where $j$ runs through a set of integers, $g_{j}(z)$ is an entire function with at most one simple zero, say $z=z_{j}$, and the convergence 
is absolute and uniform on the compact subsets of $\mathbf{C}$. Then the logarithmic derivative $\omega(z)$ of $g(z), \omega(z):=\frac{g^{\prime}(z)}{g(z)}$, admits near each simple pole $z_{k}$ a representation $\omega(z)=\frac{1}{z-z_{k}}+h_{k}(z)$, where

$$
h_{k}(z):=\sum_{j, j \neq k} \frac{g_{j}^{\prime}(z)}{g_{j}(z)}+\left(\frac{g_{k}^{\prime}(z)}{g_{k}(z)}-\frac{1}{z-z_{k}}\right)
$$

is analytic in a neighborhood of $z_{k}$. Consequently, by a simple calculation, the associated meromorphic function $\omega^{\prime}(z)+\omega^{2}(z)$ has a pole of multiplicity at most one at $z=z_{k}$, with residue $2 h_{k}\left(z_{k}\right)$. Therefore, a necessary and sufficient condition that $\omega^{\prime}(z)+\omega^{2}(z)$ be an entire function is $h_{k}\left(z_{k}\right)=0$, for every zero $z_{k}$ of $g(z)$.

For later use we record here that

$$
\omega^{\prime}(z)+\omega^{2}(z)=\sum_{j} \frac{g_{j}^{\prime \prime}(z)}{g_{j}(z)}+2 \sum_{j, k, j<k} \frac{g_{j}^{\prime}(z)}{g_{j}(z)} \frac{g_{k}^{\prime}(z)}{g_{k}(z)} .
$$

Assume now that a non-trivial entire function $f(z)$ with zeros $\left(a_{i}\right)_{i}$ and Hadamard product representation (4) is a solution to a differential equation $y^{\prime \prime}(z)=v(z) y(z)$, with $v(z)$ a polynomial function of degree $d$. Then by Wiman-Valiron theory $[\mathrm{L}$, $\rho(f)=\frac{d+2}{2}$, and clearly, $v(z)=w^{\prime}(z)+w^{2}(z)$, where $w(z):=\frac{f^{\prime}(z)}{f(z)}$ is the logarithmic derivative of $f(z)$. In particular, all the residues of $w^{\prime}(z)+w^{2}(z)$ at its potential poles vanish.

Let us specialize the above analysis to the case

$$
g_{-1}(z)=e^{q(z)}, g_{0}(z)=z^{\epsilon}, g_{i}(z)=E_{m}\left(\frac{z}{a_{i}}\right), i=1,2, \ldots
$$

Then,

$$
w(z)=q^{\prime}(z)+\frac{\epsilon}{z}+\sum_{i}\left(\frac{z}{a_{i}}\right)^{m} \frac{1}{z-a_{i}} .
$$

If $a_{k} \neq 0$ is a zero of $f(z)$, then as above

$$
\begin{aligned}
\frac{1}{2} \operatorname{Res}\left(w^{\prime}+w^{2}, z=a_{k}\right)= & q^{\prime}\left(a_{k}\right)+\frac{\epsilon}{a_{k}}+\sum_{i, i \neq k}\left(\frac{a_{k}}{a_{i}}\right)^{m} \frac{1}{a_{k}-a_{i}} \\
& +\lim _{z \rightarrow a_{k}}\left(\left(\frac{z}{a_{k}}\right)^{m} \frac{1}{z-a_{k}}-\frac{1}{z-a_{k}}\right),
\end{aligned}
$$

which yields equation (5) when $p(z)=q(z)$.

The only other zero of $f(z)$ can be $z=0$, if $\epsilon=1$, and so

$$
\frac{1}{2} \operatorname{Res}\left(w^{\prime}+w^{2}, z=0\right)= \begin{cases}q^{\prime}(0)-\sum_{i} \frac{1}{a_{i}}, & \text { if } m=0 \text { and }\left(a_{i}\right)_{i} \text { is non-empty } \\ q^{\prime}(0), & \text { otherwise }\end{cases}
$$

which in turn implies equation (6).

Conversely, assume that equations (5) and (6) hold for $p(z)=q(z)$. It is obvious that $f(z)$ is a solution to a differential equation $y^{\prime \prime}(z)=v(z) y(z)$ if and only if $w^{\prime}(z)+w^{2}(z)=v(z)$, where $w(z)=\frac{f^{\prime}(z)}{f(z)}$. As above, equations (5) and (6) show 
that $w^{\prime}(z)+w^{2}(z)$ is an entire function, and the proof will be complete if we show that actually it is a polynomial function. Equation (10) adapted to the Hadamard product representation of $f(z)$ becomes, for $m \geq 1$,

$$
\begin{aligned}
& w^{\prime}(z)+w^{2}(z)=q^{\prime}(z)^{2}+q^{\prime \prime}(z)+\sum_{i} \frac{1}{a_{i}}\left[\left(\frac{z}{a_{i}}\right)^{2 m-1}+\left(\frac{z}{a_{i}}\right)^{2 m-2}+\ldots+\left(\frac{z}{a_{i}}\right)^{m}\right. \\
& \left.+m\left(\frac{z}{a_{i}}\right)^{m-1}\right] \frac{1}{z-a_{i}}+2 \epsilon q^{\prime}(z) \frac{1}{z}+2 q^{\prime}(z) \sum_{i}\left(\frac{z}{a_{i}}\right)^{m} \frac{1}{z-a_{i}}+2 \frac{\epsilon}{z} \sum_{i}\left(\frac{z}{a_{i}}\right)^{m} \frac{1}{z-a_{i}} \\
& +2 \sum_{i, k, i<k}\left(\frac{z}{a_{i}}\right)^{m}\left(\frac{z}{a_{k}}\right)^{m} \frac{1}{z-a_{i}} \frac{1}{z-a_{k}}=q^{\prime}(z)^{2}+q^{\prime \prime}(z)+2 \epsilon \frac{q^{\prime}(z)-q^{\prime}(0)}{z} \\
& +\sum_{i} \frac{1}{a_{i}}\left[-2 m\left(\frac{z}{a_{i}}\right)^{2 m}+\left(\frac{z}{a_{i}}\right)^{2 m-1}+\ldots+\left(\frac{z}{a_{i}}\right)^{m}+m\left(\frac{z}{a_{i}}\right)^{m-1}\right] \frac{1}{z-a_{i}} \\
& +2\left(q^{\prime}(z)+\frac{\epsilon}{z}\right)\left[\sum_{i}\left(\frac{z}{a_{i}}\right)^{2 m} \frac{1}{z-a_{i}}-s_{m+1} z^{m}-s_{m+2} z^{m+1}-\cdots-s_{2 m} z^{2 m-1}\right] \\
& +2 \epsilon q^{\prime}(0) \frac{1}{z}+2 z^{2 m} \sum_{i} \frac{1}{a_{i}^{2 m}}\left[\sum_{k, k \neq i}\left(\frac{a_{i}}{a_{k}}\right)^{m} \frac{1}{a_{i}-a_{k}}+\frac{m}{a_{i}}\right] \frac{1}{z-a_{i}},
\end{aligned}
$$

where $s_{m+1}, s_{m+2}, \ldots, s_{2 m}$ are Gil' sums, namely, for $n$ an integer, $n>m, s_{n}:=$ $\sum_{i} \frac{1}{a_{i}^{n}}$. Therefore,

$$
\begin{aligned}
& w^{\prime}(z)+w^{2}(z)=q^{\prime}(z)^{2}+q^{\prime \prime}(z)+2 \epsilon \frac{q^{\prime}(z)-q^{\prime}(0)}{z}+2 \sum_{i}\left(\frac{q^{\prime}(z)-q^{\prime}\left(a_{i}\right)}{z-a_{i}}\right)\left(\frac{z}{a_{i}}\right)^{2 m} \\
& -2 q^{\prime}(z)\left(s_{m+1} z^{m}+\ldots+s_{2 m} z^{2 m-1}\right)-2 \epsilon\left(s_{m+1} z^{m-1}+\ldots+s_{2 m+1} z^{2 m-1}\right) \\
& +\sum_{i} \frac{1}{a_{i}}\left[-2 m\left(\frac{z}{a_{i}}\right)^{2 m}+\left(\frac{z}{a_{i}}\right)^{2 m-1}+\ldots+\left(\frac{z}{a_{i}}\right)^{m}+m\left(\frac{z}{a_{i}}\right)^{m-1}\right] \frac{1}{z-a_{i}} \\
& +2 \epsilon q^{\prime}(0) \frac{1}{z}+2 z^{2 m} \sum_{i} \frac{1}{a_{i}^{2 m}}\left[q^{\prime}\left(a_{i}\right)-\sum_{k, k \neq i}\left(\frac{a_{i}}{a_{k}}\right)^{m} \frac{1}{a_{k}-a_{i}}+\frac{m+\epsilon}{a_{i}}\right] \frac{1}{z-a_{i}} .
\end{aligned}
$$

The last line in the above equation vanishes, due to the hypotheses represented by the content of equations (5) and (6), and so $w^{\prime}+w^{2}$ becomes

$$
\begin{aligned}
& w^{\prime}(z)+w^{2}(z)=q^{\prime}(z)^{2}+q^{\prime \prime}(z)+2 \epsilon \frac{q^{\prime}(z)-q^{\prime}(0)}{z}+2 \sum_{i}\left(\frac{q^{\prime}(z)-q^{\prime}\left(a_{i}\right)}{z-a_{i}}\right)\left(\frac{z}{a_{i}}\right)^{2 m} \\
& -2 q^{\prime}(z)\left(s_{m+1} z^{m}+\ldots+s_{2 m} z^{2 m-1}\right)-2 \epsilon\left(s_{m+1} z^{m-1}+\ldots+s_{2 m+1} z^{2 m-1}\right) \\
& +\sum_{i} \frac{1}{a_{i}}\left[-2 m\left(\frac{z}{a_{i}}\right)^{2 m}+\left(\frac{z}{a_{i}}\right)^{2 m-1}+\ldots+\left(\frac{z}{a_{i}}\right)^{m}+m\left(\frac{z}{a_{i}}\right)^{m-1}\right] \frac{1}{z-a_{i}},
\end{aligned}
$$


which can be further simplified to read

$$
\begin{aligned}
& w^{\prime}(z)+w^{2}(z)=q^{\prime}(z)^{2}+q^{\prime \prime}(z)+2 \epsilon \frac{q^{\prime}(z)-q^{\prime}(0)}{z}+2 \sum_{i}\left(\frac{q^{\prime}(z)-q^{\prime}\left(a_{i}\right)}{z-a_{i}}\right)\left(\frac{z}{a_{i}}\right)^{2 m} \\
& -2 q^{\prime}(z)\left(s_{m+1} z^{m}+\ldots+s_{2 m} z^{2 m-1}\right)-2 \epsilon\left(s_{m+1} z^{m-1}+\ldots+s_{2 m+1} z^{2 m-1}\right) \\
& -\left(m s_{m+1} z^{m-1}+(m+1) s_{m+2} z^{m}+\ldots+(2 m-1) s_{2 m} z^{2 m-2}+2 m s_{2 m+1} z^{2 m-1}\right) .
\end{aligned}
$$

A careful inspection of equation (12) reveals an equality of two entire functions, where one, the right hand side, is a sum of a polynomial of degree at most $\max \left\{2 \operatorname{deg}\left(q^{\prime}\right), \operatorname{deg}\left(q^{\prime}\right)+2 m-1\right\}$ and an infinite sum, $\sum_{i}\left(\frac{q^{\prime}(z)-q^{\prime}\left(a_{i}\right)}{z-a_{i}}\right)\left(\frac{z}{a_{i}}\right)^{2 m}$, whose partial sums are all polynomials of degree at most $\operatorname{deg}\left(q^{\prime}\right)+2 m-1$. This is possible only if the left hand side, $w^{\prime}(z)+w^{2}(z)$, is a polynomial function.

Also, if $\operatorname{deg}\left(q^{\prime}\right) \leq m$, the infinite sum $\sum_{i}\left(\frac{q^{\prime}(z)-q^{\prime}\left(a_{i}\right)}{z-a_{i}}\right)\left(\frac{z}{a_{i}}\right)^{2 m}$ can be concretely represented as a polynomial whose coefficients depend on the coefficients of $q^{\prime}$ and on the Gil' sums $s_{m+1}, s_{m+2}, \ldots, s_{2 m}$, therefore completing an effective realization of the right hand side of equation (12) as a polynomial.

Much simpler but otherwise similar calculations show that in the case $m=0$,

$$
w(z)=q^{\prime}(z)+\frac{\epsilon}{z}+\sum_{i} \frac{1}{z-a_{i}}
$$

and then

$$
w^{\prime}(z)+w^{2}(z)=q^{\prime}(z)^{2}+q^{\prime \prime}(z)+2 \epsilon \frac{q^{\prime}(z)-q^{\prime}(0)}{z}+2 \sum_{i} \frac{q^{\prime}(z)-q^{\prime}\left(a_{i}\right)}{z-a_{i}} .
$$

Notice that when $m=0$ and $\operatorname{deg}\left(q^{\prime}\right) \geq 1, \sum_{i} \frac{q^{\prime}(z)-q^{\prime}\left(a_{i}\right)}{z-a_{i}}$ in equation (14) cannot converge unless $\left(a_{i}\right)_{i}$ is a finite set. This means that no entire function of finite order $\geq 2$, with infinitely many zeros with exponent of convergence $<1$, can be a solution to a differential equation $y^{\prime \prime}(z)=v(z) y(z), v(z)$ a polynomial or even an entire function, since then $\rho(f)=\operatorname{deg}(q) \geq 2$, and $\operatorname{so} \operatorname{deg}\left(q^{\prime}\right) \geq 1$. The proof of Theorem A is complete.

Remark. The reader may wonder why, inside the proof of Theorem A, we called the sums of like powers $s_{n}=\sum_{i} \frac{1}{a_{i}^{n}}, n>\rho(f)$, Gil' sums. This is to recognize the remarkable expressions found for them by Gil' $[\mathrm{G}$ in terms of the coefficients of the power series expansion of the entire function $\frac{f(z)}{z^{\epsilon}}$ about $z=0$. Namely, if

$$
\frac{f(z)}{z^{\epsilon}}=\sum_{i=0}^{\infty} c_{i} z^{i}, c_{0} \neq 0,
$$


then [G] for $n>\rho(f), s_{n}=\operatorname{trace}\left(A_{n}^{n}\right)$, where $A_{n}$ is the $n \times n$ matrix

$$
\left[\begin{array}{ccccc}
-\frac{c_{1}}{c_{0}} & -\frac{c_{2}}{c_{0}} & \ldots & -\frac{c_{n-1}}{c_{0}} & -\frac{c_{n}}{c_{0}} \\
1 & 0 & \ldots & 0 & 0 \\
0 & 1 & \ldots & 0 & 0 \\
\vdots & \vdots & \vdots & \vdots & \vdots \\
0 & 0 & \ldots & 1 & 0
\end{array}\right]
$$

In order to obtain a second characterization of the entire functions of finite order which are solutions to a differential equation $y^{\prime \prime}(z)=v(z) y(z), v(z)$ a polynomial function, we revisit the expression (11) of the logarithmic derivative $w(z)$ of such a function. The Laurent expansion of $w(z)$ about $z=0$, valid in the annulus $0<|z|<\left|a_{1}\right|$, is

$$
\begin{aligned}
w(z) & =\frac{\epsilon}{z}+q^{\prime}(z)-\sum_{i} \frac{z^{m}}{a_{i}^{m+1}} \frac{1}{1-\frac{z}{a_{i}}}=\frac{\epsilon}{z}+q^{\prime}(z)-\sum_{i=0}^{\infty} s_{m+i+1} z^{m+i} \\
& =\frac{\epsilon}{z}+\sum_{i=0}^{\infty} S_{i} z^{i}
\end{aligned}
$$

where $s_{i}, i=m+1, m+2, \ldots$, are the usual Gil' sums and

$$
S_{i}= \begin{cases}\frac{q^{(i+1)}(0)}{i !}, & \text { if } 0 \leq i \leq m-1, \\ \frac{q^{(i+1)}(0)}{i !}-s_{i+1}, & \text { if } i \geq m .\end{cases}
$$

Thus, for $i \geq \max \{\operatorname{deg}(q), m\}, S_{i}=-s_{i+1}$. We have $w^{\prime}(z)=-\frac{\epsilon}{z^{2}}+\sum_{i=0}^{\infty}(i+1) S_{i+1} z^{i}$ and

$$
w^{2}(z)=\frac{\epsilon^{2}}{z^{2}}+2 \epsilon \frac{S_{0}}{z}+\sum_{i=0}^{\infty}\left(2 \epsilon S_{i+1}+\sum_{k=0}^{i} S_{k} S_{i-k}\right) z^{i}
$$

Then,

$$
w^{\prime}(z)+w^{2}(z)=2 \epsilon \frac{S_{0}}{z}+\sum_{i=0}^{\infty}\left((i+1+2 \epsilon) S_{i+1}+\sum_{k=0}^{i} S_{k} S_{i-k}\right) z^{i}, \quad 0<|z|<\left|a_{1}\right|,
$$

immediately yields the following.

Theorem B. With the same notation as in Theorem A, a non-zero entire function of finite order $f(z)$ is a solution to a differential equation $y^{\prime \prime}(z)=v(z) y(z), v(z)$ a polynomial function, if and only if all the zeros of $f$ are simple,

$$
\epsilon q^{\prime}(0)= \begin{cases}\epsilon \sum_{i} \frac{1}{a_{i}}, & \text { if } m=0 \text { and }\left(a_{i}\right)_{i} \text { is non-empty, } \\ 0, & \text { otherwise }\end{cases}
$$

and all but finitely many of the Gil' sums satisfy recurrent relations

$$
s_{i+2}=\frac{1}{i+1+2 \epsilon} \sum_{k=0}^{i} S_{k} S_{i-k},
$$


where in turn $S_{k}$ is given by a relation of type (15), involving prior Gil' sums and coefficients of $q^{\prime}(z)$.

Proof. We know that a non-zero entire function $f(z)$ is a solution to a differential equation $y^{\prime \prime}(z)=v(z) y(z), v(z)$ polynomial function, if and only if all the zeros of $f$ are simple and the meromorphic function $w^{\prime}(z)+w^{2}(z), w(z)$ the logarithmic derivative of $f(z)$, is identical to the polynomial function $v(z)$. Now, in terms of the Hadamard product representation (4) of $f(z), w^{\prime}(z)+w^{2}(z)$ admits the Laurent expansion (16) about $z=0$, valid in the annulus $0<|z|<\left|a_{1}\right|$. Equation (17) is merely equivalent to the vanishing of the singular part of $w^{\prime}(z)+w^{2}(z)$, while equation (18), holding for every integer $i$ large enough, guarantees that its regular part is a polynomial. Theorem B then follows from the uniqueness of the analytic continuation of meromorphic functions.

\section{Applications}

We conclude this paper with few applications to Theorems A and B.

a) Trigonometric functions as solutions to differential equations. Theorems A and B produce interesting results even when applied to entire functions satisfying the simplest 2 nd order linear differential equations, those with constant coefficients when everything is known.

For instance, when $f(z)=\sin \pi z$, then $q^{\prime}(z) \equiv 0, \epsilon=1, a_{i}=i, i \in \mathbf{Z} \backslash\{0\}$, $m=1$, and $f(z)$ is a solution to the differential equation $y^{\prime \prime}(z)=-\pi^{2} y(z)$. In this case equation (5) becomes

$$
\frac{2}{k^{2}}=\sum_{i \in \mathbf{Z} \backslash\{0, k\}} \frac{1}{i(i-k)}, \quad k \in \mathbf{Z} \backslash\{0\},
$$

while equation (16), where $w^{\prime}(z)+w^{2}(z) \equiv-\pi^{2}$, turns out to produce the familiar values of the Riemann zeta function at the even positive integers.

If $f(z)=\cos \pi z$, then $q^{\prime}(z) \equiv 0, \epsilon=0, a_{i}=i+\frac{1}{2}, i \in \mathbf{Z}$, and $m=1$, in which case (5) becomes

$$
\frac{2}{(2 k+1)^{2}}=\sum_{i \in \mathbf{Z} \backslash\{k\}} \frac{1}{(2 i+1)(i-k)}, \quad k \in \mathbf{Z} .
$$

b) A counterexample. In relation to application a) yet quite different from it, we notice that no entire function of finite order with simple zeros $1,2, \ldots, n, \ldots$ can be a solution to a 2 nd order differential equation (1) with polynomial coefficients, a result which was also considered by Elzaidi $\mathrm{E}$.

Indeed, if that were the case, by Theorem A a polynomial function $p(z)$ would exist such that

$$
p^{\prime}(k)=\sum_{i=1, i \neq k}^{\infty} \frac{k}{i(i-k)}-\frac{1}{k}, \quad k=1,2, \ldots
$$


However, for $k \geq 2$,

$$
\begin{aligned}
\sum_{i=1, i \neq k}^{\infty} \frac{k}{i(i-k)} & =\lim _{N \rightarrow \infty, N>k} \sum_{i=1, i \neq k}^{N} \frac{k}{i(i-k)}=-\left(\frac{1}{k-1}+\frac{1}{k-2}+\ldots+1\right) \\
& +\lim _{N \rightarrow \infty, N>k}\left(1+\frac{1}{2}+\ldots+\frac{1}{N-k}-\ln (N-k)\right) \\
& -\lim _{N \rightarrow \infty}\left(1+\frac{1}{2}+\ldots+\frac{1}{N}-\ln N\right)+\frac{1}{k}+\lim _{N \rightarrow \infty, N>k} \ln \frac{N-k}{N} \\
& =\frac{1}{k}-\left(1+\frac{1}{2}+\ldots+\frac{1}{k-1}\right)+\gamma-\gamma \\
& =\frac{1}{k}-\left(1+\frac{1}{2}+\ldots+\frac{1}{k-1}\right),
\end{aligned}
$$

where $\gamma$ is the Euler constant.

Obviously, there is no polynomial function $p(z)$ such that

$$
p^{\prime}(k)=-\left(1+\frac{1}{2}+\ldots+\frac{1}{k-1}\right), \quad k=2,3, \ldots .
$$

c) Entire functions of finite order with finitely many zeros. When $f(z)$ has finitely many zeros there is no need to treat 0 separately from the non-zero zeros. In this case we represent $f(z)$ as

$$
f(z)=e^{q(z)}\left(z-z_{1}\right)\left(z-z_{2}\right) \ldots\left(z-z_{n}\right),
$$

where $z_{1}, z_{2}, \ldots, z_{n}$ are all the zeros of $f(z)$ and $q(z)$ is some polynomial function. Since $m=0$, Theorem A implies that $f(z)$ is a solution to some differential equation $y^{\prime \prime}(z)=v(z) y(z), v(z)$ a polynomial function, if and only if all the zeros of $f(z)$ are simple and

$$
q^{\prime}\left(z_{k}\right)=\sum_{i, i \neq k}^{n} \frac{1}{z_{i}-z_{k}}, \quad k=1,2, \ldots, n
$$

in which case

$$
v(z)=q^{\prime}(z)^{2}+q^{\prime \prime}(z)+2 \sum_{i=1}^{n} \frac{q^{\prime}(z)-q^{\prime}\left(z_{i}\right)}{z-z_{i}} .
$$

Notice that equation (21) implies that the polynomial $v(z)$, which must necessarily have even degree, uniquely determines the polynomial $q(z)$ up to a sign and up to an additive constant.

In fact, there are some other interesting aspects of the above analysis that one can explore: What happens if one prescribes either the zeros of $f(z)$ or the polynomial $q(z)$ together with the number $n$ of zeros of $f(z)$ ? Also, for what polynomials $v(z)$ does the equation $y^{\prime \prime}(z)=v(z) y(z)$ admit solutions of type (19)?

Prescribing the zeros $z_{1}, z_{2}, \ldots, z_{n}$ does not present a real problem, since there is a unique polynomial $i(z)$ of degree at most $n-1$ such that equation (20) is satisfied when $q^{\prime}(z)$ is replaced by $i(z)$, namely the interpolating polynomial, which at $z=z_{k}$ takes the value

$$
b_{k}:=\sum_{i, i \neq k}^{n} \frac{1}{z_{i}-z_{k}},
$$


defined by

$$
i(z)=\sum_{j=1}^{n}\left(\frac{b_{j}}{\prod_{i, i \neq j}^{n}\left(z_{j}-z_{i}\right)}\right) \prod_{i, i \neq j}^{n}\left(z-z_{i}\right) .
$$

Then the most general form of a polynomial $q^{\prime}(z)$ satisfying equation (19) is $q^{\prime}(z)=$ $\left(z-z_{1}\right) \ldots\left(z-z_{n}\right) r(z)+i(z)$, where $r(z)$ is some arbitrary polynomial function.

Now prescribing the polynomial $q(z)$ and the number $n$ of zeros of $f(z)$ in equation (19) amounts to finding $n$ distinct complex numbers $z_{1}, z_{2}, \ldots, z_{n}$ such that for a given polynomial $q(z), \operatorname{deg}(q) \geq 2$, the relations (20) hold. This is equivalent, via the content of equations (8) and (9), to finding a monic polynomial of degree $n, r(z)$, in fact $r(z)=\left(z-z_{1}\right)\left(z-z_{2}\right) \ldots\left(z-z_{n}\right)$ such that when dividing with the remainder $-2 q^{\prime}(z) r^{\prime}(z)$ by $r(z)$, we obtain the remainder $r^{\prime \prime}(z)$.

This equivalent form is in fact a terrific problem in the theory of (non-linear) polynomial systems with variable coefficients, and as such will be pursued elsewhere. It suffices to say that such a system always has finitely many solutions, being associated to a zero-dimensional algebraic variety. An intuitive argument to see that solutions always exist might be supplied along the following lines:

- Use interpolation as before to show that for a given $n$ a polynomial $q_{0}(z)$ of the same degree as $q(z)$ exists with the required properties.

- Use the homotopy $t q(z)+(1-t) q_{0}(z), 0 \leq t \leq 1$, to deform a system known to have solutions into a system desired to have solutions, and then follow the solutions through the deformation, as in $\mathrm{Wr}$. (For this purpose it can be assumed that $q(z)$ and $q_{0}(z)$ have the same leading coefficients.)

If $q(z)$ is a polynomial of even degree with real coefficients and negative leading coefficient, there is also Stieltjes' famous way [St] of finding real solutions $z_{1}$, $z_{2}, \ldots, z_{n}$, to the system (20), as critical points corresponding to the minima of the function $U\left(x_{1}, x_{2}, \ldots, x_{n}\right):=-2 \sum_{i=1}^{n} q\left(x_{i}\right)-\sum_{i<j} \ln \left(x_{i}-x_{j}\right)^{2},\left(x_{1}, x_{2}, \ldots, x_{n}\right) \in$ $\mathbf{R}^{n}, x_{i} \neq x_{j}$.

The characterization of polynomials $v(z)$ for which the equation $y^{\prime \prime}(z)=v(z) y(z)$ admits solutions of type (19) is difficult in general. Indeed, as equation (21) shows, while the monomials in $v(z)$ of sufficiently high degree are essentially determined by $q(z)$, those of low degree involve both $q(z)$ and the roots $z_{1}, z_{2}, \ldots, z_{n}$. There are, however, few instances of low-dimensional polynomials $v(z)$ when interesting characterizations can be carried out.

For instance, equation (21) implies that the quartic polynomials $v(z)$ must have the form

$$
v(z)=\alpha^{2} z^{4}+2 \alpha \beta z^{3}+\left(\beta^{2}+2 \alpha \gamma\right) z^{2}+2[(n+1) \alpha+\beta \gamma] z+\text { const },
$$

where $\alpha \neq 0, \beta, \gamma$ are arbitrary complex constants, $n$ is a non-negative integer, and for arbitrary $\alpha, \beta, \gamma$, and $n$ there are only finitely many admissible values for 'const'. Also, the sextic even polynomials must have the form

$$
v(z)=\alpha^{2} z^{6}+2 \alpha \beta z^{4}+\left[\beta^{2}-\alpha(4 n+2 \epsilon+3)\right] z^{2}+\text { const },
$$

where $\alpha, \beta, n$ are as in the quartic case and $\epsilon=0,1$. These polynomials are relevant to the spectral theory of the one-dimensional Schrödinger operators with polynomial 
potentials in that they are the only quasi-exactly solvable sextic even polynomial potentials [U, EGS1]. A more detailed account of this spectral problem will be presented in application e) below.

d) The Airy functions. They are, broadly speaking, non-trivial solutions $f(z)$ of the Airy equation $y^{\prime \prime}(z)=z y(z)$. As such, $\rho(f)=\frac{3}{2}$, so $f(z)$ must have infinitely many zeros, $\mu=\frac{3}{2}$, and $m=1$. Also, the polynomial $q(z)$ in the Hadamard product representation of $f(z)$ must have degree at most one.

We take advantage of the fact that the general solution of the Airy equation is readily available as a power series $[\mathrm{VS}]$ :

$$
\begin{aligned}
f(z) & =\sum_{k=0}^{\infty} \frac{3^{k}}{(3 k) !}\left[c_{0}\left(\frac{1}{3}\right)_{k} z^{3 k}+c_{1}\left(\frac{2}{3}\right)_{k} \frac{1}{3 k+1} z^{3 k+1}\right] \\
& =c_{0}+c_{1} z+\frac{c_{0}}{6} z^{3}+\frac{c_{1}}{12} z^{4}+\ldots,
\end{aligned}
$$

where $c_{0}, c_{1}$ are arbitrary constants, and

$$
(a)_{0}=1,(a)_{k}=\frac{\Gamma(a+k)}{\Gamma(a)}=a(a+1) \cdots(a+k-1), k>0,
$$

is the Pochhammer symbol.

Now if

$$
f(z)=e^{q_{0}+q_{1} z} z^{\epsilon} \prod_{i=1}^{\infty}\left(1-\frac{z}{a_{i}}\right) e^{z / a_{i}}
$$

is the Hadamard product representation of $f(z)$, we have basically two ways of calculating the Gil' relations: by means of the trace formula given in the Remark, via equation (22), or by means of Theorem B, via equations (15) and (16).

For instance, if $c_{0} \neq 0$ or equivalently $\epsilon=0$, the trace formula gives

$$
s_{2}=\left(\frac{c_{1}}{c_{0}}\right)^{2}, s_{3}=-\frac{1}{2}-\left(\frac{c_{1}}{c_{0}}\right)^{3}, s_{4}=\frac{1}{3} \frac{c_{1}}{c_{0}}+\left(\frac{c_{1}}{c_{0}}\right)^{4}, \ldots,
$$

while Theorem B gives

$$
s_{2}=q_{1}^{2}, s_{3}=-\frac{1}{2}-q_{1}^{3}, s_{4}=\frac{1}{3} q_{1}+q_{1}^{4}, \ldots
$$

It follows that $q_{1}=\frac{c_{1}}{c_{0}}$, and then the two sets of formulas, (24) and (25), coincide, as they should. We notice that from a computational standpoint getting the Gil' relations via Theorem $B$ is more efficient than getting them by the trace approach.

Also, if $c_{0}=0$ and $c_{1} \neq 0$ or equivalently $\epsilon=1$, both the trace formula and Theorem B give

$$
s_{2}=0, s_{3}=-\frac{1}{4}, s_{4}=0, \ldots
$$

Consequently, the polynomial $q(z)$ is constant if 0 is a zero of $f(z)$.

To conclude, we notice that for Airy functions (23) Theorem A gives

$$
q_{1}=\sum_{i, i \neq k} \frac{a_{k}}{a_{i}\left(a_{i}-a_{k}\right)}-\frac{1+\epsilon}{a_{k}}, \quad k=1,2, \ldots
$$

Other interesting identities satisfied by the zeros of the Airy functions can be found in A]. 
e) The spectral theory of the one-dimensional Schrödinger operators with polynomial potentials. The quartic anharmonic oscillator case. The spectral problem

$$
-y^{\prime \prime}(x)+v(x) y(x)=\lambda y(x), \quad x \in \mathbf{R}, y \in L^{2}(\mathbf{R}),
$$

where $v(x)$ is a real polynomial of even degree with positive leading coefficient, is known to have discrete spectrum, with all eigenvalues real and simple [BS]. Since an eigenfunction $f(x)$ must satisfy the equation $y^{\prime \prime}(x)=[v(x)-\lambda] y(x)$, we have that $f(x)$ is an entire function of order $\frac{\operatorname{deg}(v(x))+2}{2}$ (restricted to the real axis). It is also known that if the spectrum is $\lambda_{0}<\lambda_{1}<\lambda_{2}<\ldots<\lambda_{n}<\ldots \longrightarrow \infty$ and if $f_{n}(x)$ is an eigenfunction corresponding to $\lambda_{n}$, then $f_{n}(z)$ has exactly $n$ real zeros while the arguments of the complex zeros accumulate to finitely many directions, the Stokes' directions [BS, $[\mathrm{S}]$. There are also other results about the spectral decomposition of these operators D, EGS1, EGS2, T].

Now, the simplest non-trivial Schrödinger operator with polynomial potential is the quartic anharmonic oscillator, for which $v(x)=a x^{4}+b x^{2}, a>0, b \geq 0$. In this case the spectrum must be strictly positive and the complex zeros of all the eigenfunctions must be purely imaginary EGS1. Furthermore, if $f_{n}(x)$ is an eigenfunction corresponding to $\lambda_{n}$, then $f_{n}(x)$ is an even/odd function according to $n$ being even/odd, since $v(x)$ is even. Also, 0 is a zero of $f_{n}(x)$ if and only if $n$ is odd. As a result, the non-zero zeros of $f_{n}(z)$ come in pairs of opposite real/purely imaginary numbers. It turns out that in this case Theorems A and B produce some interesting identities satisfied by the zeros of any one eigenfunction and, more importantly, yield an effective way of approximating the bottom of the spectrum $\left(\lambda_{0}\right.$ and $\left.\lambda_{1}\right)$, which we proceed to describe below.

To this end fix an eigenfunction $f_{n}(x)=f(x)$ corresponding to an eigenvalue $\lambda_{n}=\lambda$ for some quartic anharmonic oscillator. Then $\rho(f)=3$ and if the zeros of $f(z)$ are

$$
\begin{cases} \pm a_{1}, \pm a_{2}, \ldots, \pm a_{j}, & \text { where } j=\left[\frac{n}{2}\right] \quad\left(a_{i}>0\right) \\ 0, & \text { if } n \text { is odd } \\ \pm \sqrt{-1} b_{1}, \pm \sqrt{-1} b_{2}, \pm \sqrt{-1} b_{3}, \ldots, & \left(b_{i}>0\right)\end{cases}
$$

then $\left(b_{i}\right)_{i=1}^{\infty}$ has exponent of convergence $\mu=3$. Up to a non-zero multiplicative constant the Hadamard product representation (4) simplifies to

$$
f(z)=e^{\alpha z^{2} / 2} z^{\epsilon} \prod_{i=1}^{[n / 2]}\left(1-\frac{z^{2}}{a_{i}^{2}}\right) e^{z^{2} / a_{i}^{2}} \prod_{i=1}^{\infty}\left(1+\frac{z^{2}}{b_{i}^{2}}\right) e^{-z^{2} / b_{i}^{2}},
$$

where a priori $\alpha$ is a complex number (depending on $n$ ), actually shown to be real below, and where, as stated earlier, $\epsilon=0$ or 1 , according to the parity of $n$. Obviously, $\left(b_{i}^{2}\right)_{i=1}^{\infty}$ has exponent of convergence $\mu=\frac{3}{2}$, and it is very tempting to link the representation (28) to the theory of Airy functions (another paper!).

The Gil' sums associated to $f(z)$ now become, for $k \geq 4$,

$$
s_{k}= \begin{cases}2 \sum_{i=1}^{[n / 2]} \frac{1}{a_{i}^{k}}+(-1)^{k / 2} 2 \sum_{i=1}^{\infty} \frac{1}{b_{i}^{k}}, & \text { if } k \text { is even, } \\ 0, & \text { if } k \text { is odd. }\end{cases}
$$


When $n=0$ or 1 , i.e., for the first two eigenvalues, when the corresponding eigenfunctions do not admit non-zero real zeros, equation (29) implies that

$$
\begin{cases}s_{k}>0, & \text { if } k \equiv 0(\bmod 4), \\ s_{k}<0, & \text { if } k \equiv 2(\bmod 4) .\end{cases}
$$

These are key inequalities which will prove decisive in an efficient approximation of the bottom of the spectrum.

The Stieltjes relations (5) given by Theorem A simplify to read in this case

$$
\left\{\begin{array}{l}
\sum_{i=1}^{\infty} \frac{a_{k}^{2}}{b_{i}^{2}\left(b_{i}^{2}+a_{k}^{2}\right)}+\sum_{i, i \neq k}^{[n / 2]} \frac{a_{k}^{2}}{a_{i}^{2}\left(a_{i}^{2}-a_{k}^{2}\right)}+\frac{1}{4 a_{k}^{2}}-\frac{3+\epsilon}{2 a_{k}^{2}}=\frac{\alpha}{2}, \quad \text { if } k=1,2, \ldots,[n / 2], \\
\sum_{i, i \neq k}^{\infty} \frac{b_{k}^{2}}{b_{i}^{2}\left(b_{i}^{2}-b_{k}^{2}\right)}+\sum_{i=1}^{[n / 2]} \frac{b_{k}^{2}}{a_{i}^{2}\left(a_{i}^{2}+b_{k}^{2}\right)}+\frac{1}{4 b_{k}^{2}}-\frac{3+\epsilon}{2 b_{k}^{2}}=-\frac{\alpha}{2}, \quad \text { if } k=1,2,3, \ldots
\end{array}\right.
$$

As far as the recurrence relations involving the Gil' sums given by Theorem B, (see also equation (16)) goes, a routine calculation shows that for any $n$ and $\lambda_{n}=\lambda$,

$$
\left\{\begin{array}{l}
s_{4}=\frac{\lambda^{2}-b}{3+2 \epsilon}, s_{6}=\frac{2 \lambda^{3}-2 b \lambda-(3+2 \epsilon) a}{(3+2 \epsilon)(5+2 \epsilon)}, \\
s_{2(i+1)}=\frac{1}{2 i+1+2 \epsilon}\left[2 \lambda s_{2 i}+\sum_{k=2}^{i-1} s_{2 k} s_{2(i+1)-2 k}\right], \quad i \geq 3
\end{array}\right.
$$

When $\epsilon=0$, equations (30) and (32) imply, via the use of $s_{4}, s_{6}$, and $s_{8}$, that

$$
\sqrt{b}<x_{8}^{0}<\lambda_{0}<x_{6}^{0},
$$

where $x_{8}^{0}$ is the largest positive zero of $17 x^{4}-22 b x^{2}-18 a x+5 b^{2}$ and $x_{6}^{0}$ is the only positive zero of $2 x^{3}-2 b x-3 a$.

Similarly, when $\epsilon=1$,

$$
\sqrt{b}<x_{8}^{1}<\lambda_{1}<x_{6}^{1}
$$

where $x_{8}^{1}$ is now the largest positive zero of $27 x^{4}-34 b x^{2}-50 a x+7 b^{2}$ and $x_{6}^{1}$ is the only positive zero of $2 x^{3}-2 b x-5 a$.

In fact, equation (32) shows that formally $s_{2 i}^{\epsilon}=s_{2 i}, i \geq 2$, is a polynomial of degree $i$ in $\lambda$ with positive leading coefficient. Replacing $\lambda$ by $x$ in $s_{2 i}^{\epsilon}$ and denoting by $x_{2 i}^{\epsilon}$ the largest positive zero of $s_{2 i}^{\epsilon}(x)$ (which always exists), it is then obvious that for $\epsilon=0,1$, equation (30) yields

$$
\lambda_{\epsilon}<x_{4 i+2}^{\epsilon}, \quad i=1,2,3, \ldots
$$

It also appears that

$$
\lambda_{\epsilon}>x_{4 i}^{\epsilon}, i=1,2,3, \ldots
$$

however, it is not obvious how to prove this in general. More intriguing is then to conjecture that

$$
\lambda_{\epsilon}=\inf _{i}\left\{x_{4 i+2}^{\epsilon}\right\}=\sup _{i}\left\{x_{4 i}^{\epsilon}\right\} .
$$


We have some numerical evidence for the above conjecture. Estimates done with Maple on the real zeros of $s_{2 i}^{\epsilon}(x)$ for $1 \leq i \leq 25$ show that for $a=1$ and $b=4$,

$$
2.158820789<\lambda_{0}<2.158820792 \text { and } 2.246526584<\lambda_{1}<2.246526587,
$$

while for $a=2$ and $b=1$,

$$
1.606005340<\lambda_{0}<1.608840883 \text { and } 1.824663890<\lambda_{1}<1.825800893 .
$$

Also, for $a=1$ and $b=0$ we have

$$
\begin{gathered}
1.050355932<\lambda_{0}<1.067325288 \text { and } 1.263014362<\lambda_{1}<1.269259101 . \\
\text { REFERENCES }
\end{gathered}
$$

[A] N. Anghel, Stieltjes-Calogero-Gil' Relations Associated to Entire Functions of Finite Orders, J. Math. Phys., 51 No. 5, 053509 (2010). MR2666987(2011f:31009)

[As] R. B. Ash, Complex Variables, Academic Press, Inc., New York (1971). MR0281883 $(43: 7597)$

[BS] F. A. Berezin, M. Shubin, The Schrödinger Equation, Kluwer, Dordrecht (1991). MR.1186643 (93i:81001)

[D] T. Duc Tai, On the Simpleness of Zeros of Stokes Multipliers, J. Differential Equations, 223, No. 2, 351-366 (2006). MR.2214939 (2006k:34242)

[E] S. M. Elzaidi, On Bank-Laine Sequences, Complex Variables Theory Appl., 38, No. 3, 201-220 (1999). MR1694317 (2000a:34170)

[EGS1] A. Eremenko, A. Gabrielov, B. Shapiro, Zeros of Eigenfunctions of Some Anharmonic Oscillators, Ann. Inst. Fourier, 58, No. 2, 603-624 (2008). MR2410384 (2009b:30007)

[EGS2] A. Eremenko, A. Gabrielov, B. Shapiro, High Energy Eigenfunctions of One-Dimensional Schrödinger Operators with Polynomial Potentials, Comput. Methods Funct. Theory, 8, No. 1-2, 513-529 (2008). MR2419492 (2009d:81100)

[F] M. Frei, Über die Lösungen linearer Differentialgleichungen mit ganzen Funktionen als Koeffizienten, Comment. Math. Helv., 35, 201-222 (1961). MR0126008 (23:A3305)

[G] M. I. Gil', Identities for Sums of Powers of Roots of Entire Functions, Compl. Var. Ellipt. Eqns., 51, No. 1, 63-68 (2006). MR2201257 (2007h:30030)

[L] I. Laine, Nevanlinna Theory and Complex Differential Equations, W. de Gruyter, Berlin, New York (1993). MR1207139 (94d:34008)

[N] J. Nikolaus, Lineare Differentialgleichungen mit gegebener ganzer Lösung, Math. Z., 103, 30-36 (1968). MR0222367 (36:5419)

[S] Y. Sibuya, Global Theory of a Second Order Linear Ordinary Differential Equation with a Polynomial Coefficient, North-Holland Publ. Co., Amsterdam (1975). MR0486867 (58:6561)

[St] T. Stieltjes, Sur Certains Polynômes qui Verifient une Équation Differentielle Linéaire du Second Ordre et sur la Théorie de Fonctions de Lamé, Acta Math., 6, 321-326 (1885). MR. 1554669

[T] A. V. Turbiner, Quasi-Exactly-Solvable Problems and sl(2) Algebra, Commun. Math. Phys., 118, 467-474 (1988). MR958807 (89j:58051)

[U] A. Ushveridze, Quasi-Exactly Solvable Models in Quantum Mechanics, Inst. Physics Publ., Bristol (1994). MR.1329549 (96i:81297)

[VS] O. Vallée, M. Soares, Airy Functions and Applications to Physics, Imperial College Press, London, 2004. MR2114198(2006c:33006)

[W] H. Wittich, Zur Theorie Linearer Differentialgleichungen im Komplexen, Ann. Acad. Sci. Fenn. Ser. A, 379, 1-18 (1966). MR0197828(33:5989)

[Wr] A. H. Wright, Finding All Solutions to a System of Polynomial Equations, Math. Comput., 44, 125-133 (1985). MR771035 (86i:12001)

Department of Mathematics, University of North Texas, Denton, Texas 76203

E-mail address: anghel@unt.edu 